\title{
The EMM and the spectral analysis of a non self-adjoint Hamiltonian on an infinite dimensional Hilbert space
}

Natalia Bebiano and João da Providência

\begin{abstract}
The Equation of Motion Method is used in the spectral analysis of a non self-adjoint bosonic Hamiltonian acting on an infinite dimensional Hilbert space. The presented operator has real eigenvalues and can be diagonalized when it is expressed in terms of pseudo-bosons, which do not behave as ordinary bosons under the adjoint transformation, but obey the Weil-Heisenberg commutation relations.
\end{abstract}

\section{Introduction}

In conventional formulations of non-relativistic quantum mechanics, the Hamiltonian operator is self-adjoint. However, certain relativistic extensions of quantum mechanics lead to the consideration of non self-adjoint Hamiltonian operators with a real spectrum. This motivated an intense research activity, both on the physical and mathematical level (see, e.g. [1, 2, 3, 4, 5, 6, 7, 8, 9] and their references).

Throughout, we shall use synonymously the terms Hermitian and self-adjoint.

Let $\mathscr{D}$ be a certain domain, dense in a Hilbert space $\mathscr{H}$, endowed with an inner product $\langle$,$\rangle . Let a, b, a^{*}, b^{*}: \mathscr{D} \rightarrow \mathscr{D}$, be bosonic operators. We recall that, conventionally, $a, b$ are said to be annihilation operators, while $a^{*}, b^{*}$ are creation operators. It is worth noticing that these operators are unbounded. Moreover, $a, b$ and of their adjoints satisfy the commutation rules (CR's),

$$
\left[a, a^{*}\right]=\left[b, b^{*}\right]=\mathbf{1}
$$

\section{Natalia Bebiano}

CMUC, University of Coimbra, Department of Mathematics, P 3001-454 Coimbra, Portugal email: bebiano@mat.uc.pt

João da Providência

CFisUC, University of Coimbra, Department of Physics, P 3004-516 Coimbra, Portugal e-mail: providencia@teor.fis.uc.pt 
where 1 is the identity operator on $\mathscr{H}$. (This means that $a a^{*} f-a^{*} a f=b b^{*} f-$ $b^{*} b f=f$ for any $f \in \mathscr{D}$.) Furthermore,

$$
\left[a, b^{*}\right]=\left[b, a^{*}\right]=\left[a^{*}, b^{*}\right]=[a, b]=0 .
$$

As it is well-known, the canonical commutation relations (1) and (2) characterize an algebra of Weil-Heisenberg (W-H). Moreover, the existence of a vector $\Phi_{0} \in \mathscr{D}$, a so-called vacuum state, satisfying,

$$
a \Phi_{0}=b \Phi_{0}=0,
$$

is postulated. The set of vectors

$$
\left\{\Phi_{m, n}=a^{* m} b^{* n} \Phi_{0}: m, n \geq 0\right\},
$$

constitutes a basis of $\mathscr{H}$, that is, every vector in $\mathscr{H}$ can be uniquely expressed in terms of this vector system, which is complete, since 0 is the only vector orthogonal to all its elements.

The main goal of this note is to investigate spectral properties of a certain non self-adjoint operator which is expressed as a quadratic combination of bosonic operators.

\section{Non self-adjoint operator and the EMM}

We are concerned with bosonic operators $a_{i}^{*}, a_{j}, i, j=1, \ldots N$, which, as usual, act on an infinite dimensional Hilbert space $\mathscr{H}$. Ordinary bosons obey the WeilHeisenberg commutation relations,

$$
\left[a_{i}, a_{j}^{*}\right]=\delta_{i j} \mathbf{1}, \quad\left[a_{i}^{*}, a_{j}^{*}\right]=0, \quad\left[a_{i}, a_{j}\right]=0, \quad i, j=1, \ldots, N,
$$

where $\delta_{i j}$ denotes the Kronecker symbol ( $\delta_{i j}$ equals 1 for $i=j$ and 0 otherwise). Let us consider the non self-adjoint Hamiltonian

$$
H=\sum_{i, j=1}^{N}\left(A_{i j} a_{i}^{*} a_{j}+\frac{1}{2} B_{i j} a_{i}^{*} a_{j}^{*}-\frac{1}{2} B_{i j} a_{i} a_{j}\right),
$$

where $A=\left(A_{i j}\right), B=\left(B_{i j}\right)$ are real symmetric matrices of size $N \times N$. In order to determine the eigenvalues of $H$, we use the equation of motion method (EMM). We investigate the condition

$$
\left[H, \sum_{i=1}^{N}\left(X_{i} a_{i}^{*}-Y_{i} a_{i}\right)\right]=\lambda \sum_{i=1}^{N}\left(X_{i} a_{i}^{*}-Y_{i} a_{i}\right),
$$


with $\lambda$ a complex parameter and $[X, Y]=X Y-Y X$ denoting, as usually, the commutator of $X$ and $Y$. From (5), we get the block matrix equation

$$
\left[\begin{array}{cc}
A & B \\
B & -A
\end{array}\right]\left[\begin{array}{l}
X \\
Y
\end{array}\right]=\lambda\left[\begin{array}{l}
X \\
Y
\end{array}\right]
$$

where $X=\left(X_{i}\right), Y=\left(Y_{i}\right)$ are column matrices with $N$ entries. Since the block matrix

$$
M=\left[\begin{array}{cc}
A & B \\
B & -A
\end{array}\right]
$$

is real symmetric, its eigenvalues $\lambda$ are real. From (6), it follows that

$$
\left[\begin{array}{cc}
A & B \\
B & -A
\end{array}\right]\left[\begin{array}{c}
Y \\
-X
\end{array}\right]=-\lambda\left[\begin{array}{c}
Y \\
-X
\end{array}\right]
$$

so, if $\lambda$ is an eigenvalue of (6), so is $-\lambda$.Thus, the eigenvalues appear in pairs of symmetric real numbers. Let us consider a set of orthogonal eigenvectors of (6). Let

$$
\left[\begin{array}{c}
X^{(r)} \\
Y^{(r)}
\end{array}\right] \text { and } \quad\left[\begin{array}{c}
Y^{(r)} \\
-X^{(r)}
\end{array}\right]
$$

be the eigenvectors corresponding to the eigenvalues $\lambda_{r}>0$ and $-\lambda_{r}$, respectively. Since they are associated to distinct eigenvalues, they are orthogonal. Orthogonality implies

$$
\begin{gathered}
X^{(r) T} X^{(s)}+Y^{(r) T} Y^{(s)}=\delta_{r s} \\
Y^{(r) T} X^{(s)}-X^{(r) T} Y^{(s)}=0 .
\end{gathered}
$$

These orthogonality relations are matricially expressed as

$$
\begin{gathered}
{\left[\begin{array}{ll}
X^{(s) T} & Y^{(s) T}
\end{array}\right]\left[\begin{array}{l}
X^{(r)} \\
Y^{(r)}
\end{array}\right]=\delta_{r s}, \quad\left[\begin{array}{ll}
X^{(s) T} & Y^{(s) T}
\end{array}\right]\left[\begin{array}{c}
Y^{(r)} \\
-X^{(r)}
\end{array}\right]=0,} \\
{\left[\begin{array}{ll}
Y^{(s) T} & -X^{(s) T}
\end{array}\right]\left[\begin{array}{c}
X^{(r)} \\
Y^{(r)}
\end{array}\right]=0, \quad\left[\begin{array}{ll}
Y^{(s) T} & -X^{(s) T}
\end{array}\right]\left[\begin{array}{c}
Y^{(r)} \\
-X^{(r)}
\end{array}\right]=\delta_{r s},}
\end{gathered}
$$

or, compactly, as

$$
\left[\begin{array}{cc}
\mathscr{X} & -\mathscr{Y} \\
\mathscr{Y} & \mathscr{X}
\end{array}\right]^{T}\left[\begin{array}{cc}
\mathscr{X} & -\mathscr{Y} \\
\mathscr{Y} & \mathscr{X}
\end{array}\right]=I_{2 N},
$$

where $I_{2 N}$ is the $2 N \times 2 N$ identity matrix and

$$
\mathscr{X}=\left[\begin{array}{lll}
X^{(1)} & \ldots & X^{(N)}
\end{array}\right], \quad \mathscr{Y}=\left[\begin{array}{lll}
Y^{(1)} & \ldots & Y^{(N)}
\end{array}\right] \in M_{N},
$$

the algebra of $N \times N$ real matrices. The matrix 


$$
\left[\begin{array}{cc}
\mathscr{X} & \mathscr{Y} \\
-\mathscr{Y} & \mathscr{X}
\end{array}\right]=\exp \left[\begin{array}{cc}
S & T \\
-T & S
\end{array}\right], \quad S=-S^{T}, \quad T=T^{T},
$$

belongs to a certain subgroup of the real orthogonal group and the matrix

$$
\left[\begin{array}{cc}
S & T \\
-T & S
\end{array}\right]
$$

belongs to a certain sub-algebra of the algebra of the real skew-symmetric matrices. Consider the pseudo-bosons defined as

$$
c_{r}^{\ddagger}=\sum_{i=1}^{N}\left(X_{i}^{(r)} a_{i}^{*}-Y_{i}^{(r)} a_{i}\right), \quad c_{r}=\sum_{i=1}^{N}\left(Y_{i}^{(r)} a_{i}^{*}+X_{i}^{(r)} a_{i}\right), \quad r, s=1, \ldots, N .
$$

Although $c_{r}^{\dagger} \neq c_{r}^{*}$, pseudo-bosons obey the Weil-Heisenberg commutation relations,

$$
\left[c_{r}, c_{s}^{\ddagger}\right]=\delta_{r s} \mathbf{1}, \quad\left[c_{r}^{\ddagger}, c_{s}^{\ddagger}\right]=0, \quad\left[c_{r}, c_{s}\right]=0, \quad r, s=1, \ldots, N,
$$

where 1 is the identity on $\mathscr{H}$. The expressions (7) may be inverted, using the orthogonality relations, as

$$
a_{i}^{*}=\sum_{r=1}^{N}\left(X_{i}^{(r)} c_{r}^{\ddagger}+Y_{i}^{(r)} c_{r}\right), \quad a_{i}=\sum_{r=1}^{N}\left(-Y_{i}^{(r)} c_{r}^{\ddagger}+X_{i}^{(r)} c_{r}\right) .
$$

From these expressions, we obtain

$$
H=\sum_{i=1}^{N} \sum_{r=1}^{N} \lambda_{r} Y_{i}^{(r) 2} \mathbf{1}+\sum_{r=1}^{N} \lambda_{r} c_{r}^{\ddagger} c_{r} .
$$

The eigenvectors of $H$ are of the form

$$
\Psi_{n_{1}, \ldots, n_{N}}=c^{\ddagger n_{1}} \cdots c^{\ddagger n_{N}} \Psi_{0}
$$

where $\Psi_{0}$ is such that

$$
c_{1} \Psi_{0}=0, \ldots, c_{N} \Psi_{0}=0,
$$

and the respective eigenvalues are of the form

$$
E_{n_{1}, \ldots, n_{N}}=\sum_{i=1}^{N} \sum_{r=1}^{N} \lambda_{r} Y_{i}^{(r) 2}+\sum_{r=1}^{N} n_{r} \lambda_{r}
$$

so that

$$
H \Psi_{n_{1}, \ldots, n_{N}}=E_{n_{1}, \ldots, n_{N}} \Psi_{n_{1}, \ldots, n_{N}} .
$$

Similarly, the eigenvectors of 


$$
H^{*}=\sum_{i, j=1}^{N}\left(A_{i j} a_{i}^{*} a_{j}-\frac{1}{2} B_{i j} a_{i}^{*} a_{j}^{*}+\frac{1}{2} B_{i j} a_{i} a_{j}\right) .
$$

are given by

$$
\Psi_{n_{1}, \ldots, n_{N}}^{\prime}=c^{* n_{1}} \cdots c^{* n_{N}} \Psi_{0}^{\prime}
$$

where $\Psi_{0}^{\prime}$ is such that

$$
c_{1}^{\ddagger *} \Psi_{0}^{\prime}=0, \ldots, c_{N}^{\ddagger *} \Psi_{0}^{\prime}=0 .
$$

The eigenvalues of $H$ and $H^{*}$ coincide. The associated eigenvector systems are biorthogonal:

$$
\left\langle\Psi_{n_{1}, \ldots, n_{N}}^{\prime}, \Psi_{m_{1}, \ldots, m_{N}}\right\rangle=n_{1} ! \cdots n_{N} ! \delta n_{1} m_{1} \cdots \delta n_{N} m_{N}\left\langle\Psi_{0}^{\prime}, \Psi_{0}\right\rangle
$$

Next, the existence of the vacua vectors $\Psi_{0}$ and $\Psi_{0}^{\prime}$ is discussed. The real skewsymmetric matrix

$$
\left[\begin{array}{cc}
S & T \\
-T & S
\end{array}\right]
$$

induces the operator

$$
\mathscr{S}=-\frac{1}{2} \sum_{i, j=1}^{N}\left(s_{i j}\left(a_{i}^{*} a_{j}+a_{j} a_{i}^{*}\right)+t_{i j} a_{i}^{*} a_{j}^{*}+t_{i j} a_{i} a_{j}\right), \quad\left(s_{i j}\right)=S,\left(t_{i j}\right)=T
$$

which satisfies

$$
\begin{aligned}
& \mathrm{e}^{\mathscr{S}} a_{r}^{*} \mathrm{e}^{-\mathscr{S}}=c_{r}^{\ddagger}=\sum_{i=1}^{N}\left(X_{i}^{(r)} a_{i}^{*}-Y_{i}^{(r)} a_{i}\right), \\
& \mathrm{e}^{\mathscr{S}} a_{r} \mathrm{e}^{-\mathscr{S}}=c_{r}=\sum_{i=1}^{N}\left(Y_{i}^{(r)} a_{i}^{*}+X_{i}^{(r)} a_{i}\right), \quad r, s=1, \ldots, N .
\end{aligned}
$$

By definition, we shall consider

$$
\mathscr{D}=\left\{\sum_{n_{1}, \ldots, n_{N}} z_{n_{1}, \ldots, N} a^{* n_{1}} \cdots a^{* n_{N}} \Phi_{0}: z_{n_{1}, \ldots, N} \in \mathbb{C}, n_{i} \geq 0\right\},
$$

where the sum is finite. Some considerations are in order. We observe that $a_{j}^{*}$ and $a_{j}$ map $\mathscr{D}$ into $\mathscr{D}$ and that $\mathscr{S}^{n} \Phi_{0} \in \mathscr{D}, 0 \leq n \in \mathbf{Z}$, where $\Phi_{0} \in \mathscr{D}$ is the vacuum of the operators $a_{i}$, i.e. a vector such that

$$
a_{1} \Phi_{0}=0, \ldots, a_{N} \Phi_{0}=0
$$

whose existence is postulated. It follows that

$$
\Psi_{0}=\mathrm{e}^{\mathscr{S}} \Phi_{0}, \quad \Psi_{0}^{\prime}=\mathrm{e}^{-S} \Phi_{0} .
$$


Consider the series expansion

$$
\sum_{n=0}^{\infty} \frac{(\gamma \mathscr{S})^{n}}{n !} \Phi_{0}, \quad \gamma= \pm 1
$$

The following question naturally arises: does it converge? Can we ensure that $\Psi_{0}$ belongs to $\mathscr{H}$ ? This point must be investigated on a case by case basis. In the next section it is considered for a specific example in which these questions are affirmatively answered.

The following remark is in order. The spectral analysis of a non self-adjoint Hamiltonian quadratic in bosonic operators should be preceded by the spectral analysis of its self-adjoint part. Recall that a self-adjoint Hamiltonian quadratic in bosonic operators may not have a real spectrum, as is the case of the self-adjoint operator

$$
x^{2}+\frac{\mathrm{d}^{2}}{\mathrm{~d} x^{2}}: \mathscr{D} \rightarrow \mathscr{D}
$$

which does not have real eigenvalues. Indeed, for instance,

$$
\left(x^{2}+\frac{\mathrm{d}^{2}}{\mathrm{~d} x^{2}}\right) \mathrm{e}^{i x^{2} / 2}=i \mathrm{e}^{i x^{2} / 2} .
$$

Notice that $\mathrm{e}^{i x^{2} / 2} \notin \mathscr{H}$, because $\left\langle\mathrm{e}^{i x^{2} / 2}, \mathrm{e}^{i x^{2} / 2}\right\rangle=+\infty$. In general, a non self-adjoint Hamiltonian may have a real spectrum and a system of eigenvectors only if the spectrum of its self-adjoint part is real. Only then its system of eigenvectors will be biorthogonal to the system of eigenvectors of the adjoint Hamiltonian.

\section{Example}

As a simple illustrative example of application of the EMM developed in the previous section, we consider the Hamiltonian in (4) for case $N$ even and with

$$
A=\bigoplus_{i=1}^{N / 2} A_{i}, \quad B=\bigoplus_{i=1}^{N / 2} B_{i}
$$

where

$$
A_{i}=\left[\begin{array}{cc}
\alpha_{i} & 0 \\
0 & \alpha_{i}
\end{array}\right], \quad B_{i}=\left[\begin{array}{cc}
0 & \beta_{i} \\
\beta_{i} & 0
\end{array}\right], \quad \alpha_{i}, \beta_{i}>0 .
$$

The EMM condition $[H, Z]=\lambda Z$, for

$$
Z=X_{1} a_{1}^{*}+X_{2} a_{2}^{*}-Y_{1} a_{1}-Y_{2} a_{2}+\ldots+X_{N-1} a_{N-1}^{*}+X_{N} a_{N}^{*}-Y_{N-1} a_{N-1}-Y_{N} a_{N}
$$

by this order, leads to the real symmetric matrix 


$$
M=\bigoplus_{i=1}^{N / 2}\left[\begin{array}{cccc}
\alpha_{i} & 0 & 0 & \beta_{i} \\
0 & \alpha_{i} & \beta_{i} & 0 \\
0 & \beta_{i} & -\alpha_{i} & 0 \\
\beta_{i} & 0 & 0 & -\alpha_{i}
\end{array}\right]
$$

whose positive eigenvalues are as follows

$$
\lambda_{1}=\lambda_{2}=\sqrt{\alpha_{1}^{2}+\beta_{1}^{2}}, \lambda_{3}=\lambda_{4}=\sqrt{\alpha_{2}^{2}+\beta_{2}^{2}}, \ldots, \lambda_{N-1}=\lambda_{N}=\sqrt{\alpha_{N / 2}^{2}+\beta_{N / 2}^{2}} .
$$

Notice that $\alpha_{i}$ and $\pm \beta_{i}$ are the eigenvalues of the blocks $A_{i}$ and $B_{i}$, respectively. In terms of pseudo-bosonic operators, which are determined by the eigenvectors of (8) associated to positive and negative eigenvalues, $H$ is given by

$$
H=\sum_{r=1}^{N / 2}\left(\sqrt{\alpha_{r}^{2}+\beta_{r}^{2}}-\alpha_{r}+\sqrt{\alpha_{r}^{2}+\beta_{r}^{2}}\left(c_{2 r-1}^{\ddagger} c_{2 r-1}+c_{2 r}^{\ddagger} c_{2 r}\right)\right) \text {. }
$$

For

$$
\mathscr{S}=\theta_{1}\left(a_{1}^{*} a_{2}^{*}+a_{1} a_{2}\right)+\theta_{2}\left(a_{3}^{*} a_{4}^{*}+a_{3} a_{4}\right)+\ldots+\theta_{N / 2}\left(a_{N-1}^{*} a_{N}^{*}+a_{N-1} a_{N}\right),
$$

where $-\pi / 2 \leq \theta_{i} \leq \pi / 2, i=1, \ldots, N / 2$, we obtain

$$
\begin{aligned}
& \mathrm{e}^{\mathscr{S}}\left(\sqrt{\alpha_{1}^{2}+\beta_{1}^{2}}\left(a_{1}^{*} a_{1}+a_{2} a_{2}^{*}\right)+\ldots+\sqrt{\alpha_{N / 2}^{2}+\beta_{N / 2}^{2}}\left(a_{N-1}^{*} a_{N-1}+a_{N} a_{N}^{*}\right)\right) \mathrm{e}^{-\mathscr{S}} \\
& =\sqrt{\alpha_{1}^{2}+\beta_{1}^{2}} \cos 2 \theta_{1}\left(a_{1}^{*} a_{1}+a_{2} a_{2}^{*}\right)+\ldots+\sqrt{\alpha_{N / 2}^{2}+\beta_{N / 2}^{2}} \cos 2 \theta_{N / 2}\left(a_{N-1}^{*} a_{N-1}+a_{N} a_{N}^{*}\right) \\
& +\sqrt{\alpha_{1}^{2}+\beta_{1}^{2}} \sin 2 \theta_{1}\left(a_{1} a_{2}-a_{1}^{*} a_{2}^{*}\right)+\ldots+\sqrt{\alpha_{N / 2}^{2}+\beta_{N / 2}^{2}} \sin 2 \theta_{N / 2}\left(a_{N-1} a_{N}-a_{N-1}^{*} a_{N}^{*}\right)
\end{aligned}
$$

Taking

$$
\tan 2 \theta_{1}=-\frac{\beta_{2}}{\alpha_{2}}, \ldots, \tan 2 \theta_{N / 2}=-\frac{\beta_{N / 2}}{\alpha_{N / 2}}
$$

we find

$$
\begin{aligned}
& \mathrm{e}^{\mathscr{S}}\left(\sqrt{\alpha_{1}^{2}+\beta_{1}^{2}}\left(a_{1}^{*} a_{1}+a_{2} a_{2}^{*}\right)+\ldots+\sqrt{\alpha_{N / 2}^{2}+\beta_{N / 2}^{2}}\left(a_{N-1}^{*} a_{N-1}+a_{N} a_{N}^{*}\right)\right) \mathrm{e}^{-\mathscr{S}} \\
& =\alpha_{1}\left(a_{1}^{*} a_{1}+a_{2} a_{2}^{*}\right)+\beta_{1}\left(a_{1}^{*} a_{2}^{*}-a_{1} a_{2}\right)+\ldots \\
& +\alpha_{N / 2}\left(a_{N-1}^{*} a_{N-1}+a_{N} a_{N}^{*}\right)+\beta_{N / 2}\left(a_{N-1}^{*} a_{N}^{*}-a_{N-1} a_{N}\right) .
\end{aligned}
$$

We have shown that the desired transformation is given by $e^{\mathscr{S}}$.

Recall that $\Phi_{0} \in \mathscr{D}$ is the vacuum of the operators $a_{i}, i=1 \ldots, N$. Next we prove that $\left\langle\mathrm{e}^{\mathscr{S}} \Phi_{0}, \mathrm{e}^{\mathscr{S}} \Phi_{0}\right\rangle<\infty$, so that the groundstate eigenvector of $H$ is $\mathrm{e}^{\mathscr{S}} \Phi_{0} \in$ span $\mathscr{D}=\mathscr{H}$. Indeed, it may be checked that the vector $\mathrm{e}^{\mathscr{S}} \Phi_{0}$ and the vector

$$
\Xi_{0}=\exp \left(\tan \theta_{1} a_{1}^{*} a_{2}^{*}+\ldots+\tan \theta_{N / 2} a_{N-1}^{*} a_{N}^{*}\right) \Phi_{0}
$$




$$
=\sum_{n_{1}=1}^{\infty} \ldots \sum_{n_{N / 2}=1}^{\infty} \frac{\tan ^{n_{1}} \theta_{1}}{n_{1} !} \cdots \frac{\tan ^{n_{N / 2}} \theta_{N / 2}}{n_{N / 2} !} a_{1}^{* n_{1}} a_{2}^{* n_{1}} \cdots a_{N-1}^{* n_{N / 2}} a_{N}^{* n_{N / 2}} \Phi_{0}
$$

may differ only by a numerical factor. Notice that

$$
c_{i} \mathrm{e}^{\mathscr{S}} \Phi_{0}=c_{i} \Xi_{0}=0, i=1, \ldots, N .
$$

Actually, $\mathrm{e}^{\mathscr{S}} \Phi_{0}$ reduces to $\Xi_{0}$ by a convenient rearrangement of the series. Now,

$$
\tan \theta_{i}=\alpha_{i} / \beta_{i}-\sqrt{1+\left(\alpha_{i} / \beta_{i}\right)^{2}}
$$

so that $\tan ^{2} \theta_{i}<1$, and

$$
\left\langle\Xi_{0}, \Xi_{0}\right\rangle=\prod_{i=1}^{N / 2} \sum_{n=0}^{\infty} \tan ^{2 n} \theta_{i}=\prod_{i=1}^{N / 2}\left(1-\tan ^{2} \theta_{i}\right)^{-1}<\infty .
$$

It follows that $\Xi_{0} \in \mathscr{H}$. We observe that the geometric series $\sum_{n=0}^{\infty} \tan ^{2 n} \theta_{i}$ with ratio $\tan ^{2} \theta_{i}<1$, converges in the interior of the unitary disc.

\section{Discussion}

In Section 2, a non self-adjoint Hamiltonian, which is expressed as a quadratic combination of bosonic operators, is investigated. Its eigenvalues and eigenvectors have been determined with the help of a real symmetric matrix $M$ of size $2 N \times 2 N$, where $N$ is the number of bosonic states, that is determined by the EMM. The investigated Hamiltonian has a system of eigenvectors expressed in terms of the creation and annihilation operators of pseudo-bosons, which is biorthogonal to the system of eigenvectors of the adjoint Hamiltonian, constructed in terms of pseudo-bosonic operators acting on the associated vacuum state.

\section{Acknowledgments}

The authors are grateful to the Referee for valuable comments.

This work was partially supported by the Centre for Mathematics of the University of Coimbra - UID/MAT/00324/2013, funded by the Portuguese Government through FCT/MEC and co-funded by the European Regional Development Fund through the Partnership Agreement PT2020. 


\section{References}

1. F. Bagarello, Construction of pseudo-bosons systems, J. Math. Phys. 51 (2010) 023531; Pseudo-bosons, so far, Reports on Mathematical Physics, 68 (2011) 175-210; More mathematics for pseudo-bosons, J. Math. Phys, 54 (2013) 063512.

2. F. Bagarello, F. Gargano, D. Volpe, $\mathscr{D}$-deformed harmonic oscillators, International Journal of Theoretical Physics 01/2015.

3. N. Bebiano, J. da Providência and JP. da Providência, Hamiltonians expressed in terms of bosonic operators and their spectra, submitted.

4. J. da Providência, N. Bebiano and JP. da Providência, Non Hermitian operators with real spectra in Quantum Mechanics, Brazilian Journal of Physics, 41 (2011) 78-85.

5. F.G. Scholtz, H.B. Geyer and F.J.W. Hahne, Quasi-Hermitian operators in quantum mechanics and the variational principle, Ann. Phys. NY 213 (1992) 74.

6. C.M. Bender and S. Boettcher, Real Spectra in Non-Hermitian Hamiltonians Having PT Symmetry , Phys. Rev. Lett., 80 (1998) 5243-5246, C.M. Bender, D.C. Brody and H.F. Jones, Complex Extension of Quantum Mechanics, Phys. Rev. Lett, 89 (2002) 27041.

7. A. Gonzaléz Lopéz and T. Tanaka, Nonlinear pseudo-supersymmetry in the framework of $N$-fold supersymmetry, J. Phys. A: Math. Gen. 39 (2006) 3715-23; T. Tanaka, Preprint quantph/0603075; T. Tanaka, J. Phys. A. Math. Gen. 39 (2006) L369-L376.

8. M. Znojil, Should PT Symmetric Quantum Mechanics Be Interpreted as Nonlinear?, J. Nonlin. Math. Phys., 9 (2002) 122-123.

9. A Mostafazadeh, Exact PT-symmetry is equivalent to Hermiticity, J. Phys. A: Math. Gen. 36 (2003) 7081; Complex Extension of Quantum Mechanics, J. Math. Phys. 46 (2005) 102108; Delta-function potential with a complex coupling, J. Phys. A: Math. Gen. 39 (2006) 13495; Pseudo-Hermicity and generalized PT- and CPT- symmetries, J. Math. Phys. 44 (2003) 974; arXiv:quant-ph/060173v2. 